Research Paper

\title{
INTEGRATION OF CLIMATE VULNERABILITY ASSESSMENT OF CIVIL SOCIETY ORGANIZATIONS INTO NATIONAL ADAPTATION PLAN (NAP) IN VIETNAM
}

\author{
Hoang Thi Ngoc Ha ${ }^{1}$, Nghiem Thi Phuong Tuyen ${ }^{2}$, Bui Thi Kim Oanh ${ }^{3}$
}

\section{ARTICLE HISTORY}

Received: October 08, 2019 Accepted: November 12, 2019

Publish on: December 25, 2019

\section{ABSTRACT}

Civil society organizations (CSOs) in Vietnam have been making significant contributions to climate change adaptation at both local and national levels. CSOs have been applying various methods in climate vulnerability assessment (CVA) to develop practical adaptive measures, with the more commonly used ones being "community-based", "participatory", and so on. The CVA methods that most CSOs have applied to climate change studies and projects in more than 30 provinces and cities include: HVCA, PVCA, $C V C A$ and $V A-E B A$. The process and effectiveness of each method is different, depending on the technical capacity and support resources. Overall, the CVA results obtained are not only an important input for various adaptation programs, projects and models in localities but also a major contribution of non-governmental organizations to the National Adaptation Plan. This paper aims to provide evidence of climate vulnerability assessment for adaptation projects that have been implemented by CSO in the last 10 years as well as feedback of vulnerable communities and policy recommendations.

Keywords: Civil society organizations (CSOs), Climate vulnerability assessment (CVA), Climate change, National Adaptation Plan (NAP).

\section{Introduction}

Climate change is becoming more intense globally, causing ever greater damage to the environment, and the natural resources and the socioeconomic aspects of human communities. In October 2018, as nominated by the Intergovernmental Panel on Climate Change (IPCC), Vietnam was selected to be the very first nation to present the Special Report on Global Warming of $1,5^{\circ}$. The "Paris Agreement on Climate Change" in 2015 also showed the countries' determination to limit the increase of Earth's temperature to less than $2^{\circ} \mathrm{C}$, or even to less than $1.5^{\circ} \mathrm{C}$ by the end of the century (compared to pre-industrial period) (TTCP, 2016). The Government and people of Vietnam have made great efforts in dealing with climate change, reflected in local policies and actions. Revisions and amendments of the Nationally Determined Contributions (NDC) and the National Adaptation Plan (NAP) are also underway. Priority and longterm adaptation measures will be identified by NAP to increase national resilience to climate change impacts. This is an important tool that contributes to reducing Climate Change Vulnerability, building community and ecosystem adaptive capacity (UNFCCC, 2015). The development of NAP requires the involvement

$\triangle$ HOANG THI NGOC HA

Corresponding author: hahoang.ecode@gmail.com

${ }^{1}$ Center for Eco-Community Development (ECODE), Vietnam

${ }^{2}$ VNU-Central Institute for Natural Resources and Environmental Studies (CRES) 
of regulatory agencies, scientists, communities and social organizations. The process of adaptive with climate change in general and the development of NAP in particular has recognized the contributions of social and non-governmental organizations in Vietnam. CSOs have developed hundreds of initiatives and activities in order to respond to climate change and to reduce disaster risks across different fields and localities with a greater focus on vulnerable groups. By implementing proactive, innovative and transparent operations, CSOs have achieved tangible results in enhancing the awareness of adaptation and resilience capacity of communities and stakeholders. Outstanding among them is the Climate Vulnerability Assessment (CVA) approach adopted with the engagement of vulnerable communities. There is a need for CVA implementation to identify objects, sectors, and areas susceptible to climate change impacts in the present and future and assess their vulnerability status and level so that effective adaptation measures can be defined and adopted in order to enhance resilience and foster sustainable development (Füssel and Klein, 2006).

\section{Methodology}

The research was conducted on the basis of reviewing and evaluating the results of the implementation of studies and projects of more than 20 representative CSOs in Vietnam, who have conducted climate vulnerability assessment at community level over the past 20 years. The Center for Eco-community Development (ECODE) conducted this research with the technical and financial support of the FriedrichEbert-Stiftung (FES), Germany and Climate Change Working Group in Vietnam (CCWG).

This research adopts the users' perception of satisfaction approach. The authors examine the perceptions and feedback of CSOs that imple- mented CVAs in their programs and projects in more than 30 provinces in 7 priority sectors of NAP. The main used methodologies are secondary research, focus group discussions, interviews with information providers/major stakeholders, baseline survey and consult expert. Questionnaires with pre-defined questions were sent to sample CSOs to fill out and in-depth interviews were conducted with staff of CC related-government offices and representatives of lead CSOs in the CC sector. The purpose is to clarify the awareness and capacity of CSOs as well as the results of their CVA implementation. The research also aimed to draw lesson learn from stakeholders who have been contributing to the suitability, feasibility and effectiveness of the CVA methodology that CSOs have applied. By engaging various stakeholders, the research was able to capture voices and feedback from vulnerable communities.

The research has three main objectives: (1) conduct a baseline survey of CVA methods and results that CCWG members have conducted in priority sectors of Vietnam's NAP process; (2) Analyze and identify the most common and appropriate CVA methods with insights from CSO perspectives; (3) Identify adaptation measures and models from grassroots $\mathrm{CSO}$ projects that need to be considered by government agencies as they develop specific implementation plans on adaptation options in the this priority sectors.

\section{Results}

A review of CSO action plans, programs and projects shows that CSOs in Vietnam have conducted CVAs to meet specific objectives: i) Define and describe local climate hazards and their impacts and potential impacts; ii) Identify objects, areas, services and functions that are most vulnerable to current climate hazards and subject to high risks in the future due to climate change; iii) Identify direct impacts of climate 
change on the most vulnerable groups of people, infrastructure and areas; understand how indirectly impacts affect others; iv) Assess people's capacities and needs for adaptation; Assess the way services and ecosystem functions can respond to on-going pressure; and v) Provide information, develop appropriate intervention models, and enhance adaptive capacity for localities and people. CSOs emphasize that the core and most important factor of CVAs is that CVA results and the process of CVA implementation must be connected to development of adaptation models to mitigate vulnerability. CVA results enable CSOs to define "gaps" and design adaptation models and activities which can "fill the gaps".

\section{Most common CVA methods implemented by CSOs in Vietnam}

CSOs' initiatives and solutions to support community adaptation were implemented in many provinces and have achieved positive results. Many adaptation models have been created based on the results of Climate Vulnerability Assessments (CVAs). CVA results not only facilitate the development of adaptation strategies, plans, and measures at provincial level but also make great contributions to macro and long-term adaptation policies.

The integration of CSO's CVA approach and results into the NAP helps to clarify the CSOs' roles and contribution (in concurrence with government activities) and institutionalize their recommendations in national adaptation policies. Assessment results show that poor and disadvantaged people are most susceptible to impacts of climate change. Climate vulnerability assessments are of great importance in identifying vulnerable subjects, areas, and sectors and in finding appropriate adaptation strategies and measures to reduce risks and leverage development opportunities.

Two major approaches of CVA implementation observed in Vietnam are: 1) the top-down approach that mainly done by government agencies and research institutions; and 2) the bottom- up community-based approach. This approach is participatory, providing community's perspective and allowing space for practical vulnerability information and feedback. The CVA methods described below are the most common adopted by CSOs in many of their programs and projects.

- HVCA - Hazard Vulnerability Capacity Assessment

Nearly $71 \%$ of the surveyed CSOs claimed that they have used this approach. HVCA is a prevailing CVA method whose development and adoption are pioneered by the Vietnam Red Cross from the late 1990s, assessing the hazards of natural disasters and climates so as to offer solutions to minimize risks and ensure safety for the community. HVCA puts emphasis on five aspects: (1) Livelihoods, (2) Basic living standards, (3) Self-protection, (4) Social protection, and (5) Civil society organizations (Le, 2017). HVCA's major components are designed to identify exposure levels, locations, vulnerable groups, and adaptive capacity of vulnerable groups. Measures following the implementation of HVCA tend to be developing plans for community safety enhancement and risk reduction, such as establishing early warning systems and building shelters. Therefore, HVCA is suitable for projects that focus on community safety. Qualitative and less-mentioned long-term climate risks are the drawbacks of this approach.

\section{- PVCA-Participatory Vulnerability Capac- ity Assessment}

$64 \%$ of the interviewed CSOs used this method. PVCA is a combination of tools to help communities develop and implement plans to enhance resilience to climate change. This method is based on the participation of community members to identify and classify climate hazards as well as to analyze resources (capacities) and local availability opportunities in order to reduce risks. PVCA is more about disaster risk reduction and emphasizes the participation of vulnerable groups (the poor, children and people with disabilities). PVCA is very useful for poverty reduction and gender equality related programs. 


\section{- CVCA - Climate Vulnerability Capacity Analysis}

$35 \%$ of CSOs and experts have used CVCA for the last 6 - 8 years, mainly for natural resource-related sectors, such as agriculture, water, mangrove forests, and so on. The key components of CVCAs are exposures, sensitivity, potential impacts, and adaptive capacity. CVCA enables identification of practical solutions or strategies, boosting community-based adaptation measures. As an open tool, its results depend on community discussions to setpriorities before developing adaptation plans, models, and solutions. CARE is a lead organization in CVCA application in Vietnam. CARE joined in a mission with UNDP-GCF Project to combine PVA and CVCA into a less sophisticated method which can be applied across NAP special sectors. GCF is now extending the application of this method.

- VA-EbA - Vulnerability AssessmentEcosystem-based Adaptation/EbA

This approach has been strongly recommended in Water, Land, Biodiversity and Transpotation sectors, which are significantly affected by the quality of the ecosystem. It is an evolution from the Ecosystem-based Adaptation (EbA) approach proposed by the UN's Convention on Biological Diversity (1992). Currently, many researchers in Vietnamare extending the application of this approach to their research. EbA proves to be helpful in the pursuit of a fair balance between the protection and the rational use of biodiversity, enabling vulnerability mitigation, resilience to climate change, and sustainable development (Hoang and Truong, 2016). VA-EbA provides detailed assessments of factors contributing to vulnerability, boasts a harmonious combination of quantitative and qualitative methods; prudently examines potential natural risks (ecosystems), conducts assessments at both the provincial and locallevel, and promotes involvement and coordination of provincial specialized officials and local people. VA-EbA-based adaptation measures will meet three objectives (socio-economy, environment, and biodiversity preservation) and highly accurate (Doswald et al., 2011). However, VA-EbA requires years of meteorological, climate and natural disaster data as well as relatively sophisticated vulnerability treatments and mapping techniques, which are mostly appropriate for the assessments bya key technical expert. This is a difficulty for the expansion and transfer of CVA at the local level.

In addition, CSOs and other agencies also use different methods depending on their objectives, targets and evaluation scale, such as: Urban Vulnerability Index (UVI), Livelihood Vulnerability Index (LVI), Social Vulnerability Index (SVI), Ecological Risk Assessment (ERA), Simulation methods, IPCC climate impacts assessment framework, Flowing Forward, Oxfam's Vulnerability and Risk Assessment (VRA) and CVA of agriculture value chains. The most popular tools used in Participatory Rapid Appraisal (PRA) by CSOs in CVA are: Seasonal calendars and disaster brief history, Community risk maps (hazard maps), Problem tree, Transect survey and analysis, Venn diagram and Group discussion.

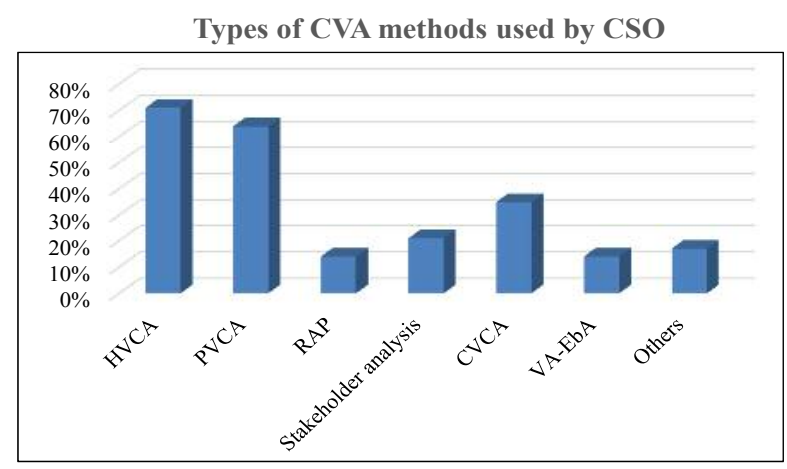

Fig. 1. CVA methods

According to the findings of the research: CSOs implemented disaster risk and climate change-related programs and projects; supported communities in livelihoods development with the application of CVA; and each organization applied several tools. The Center for Rural Development in Central Vietnam (CRD) and WWF Vietnam used a variety of methods. CSOs that mainly working on climate change and disaster risk reduction in Vietnam, like CARE, Oxfarm, World Vision, Plan, SNV, SRD and MCD, tend 
to apply HVCA and PVCA. Meanwhile, WWF and GIZ are pioneers in the application of VAEbA with an attention the vulnerability of ecosystems in relation to livelihoods. It should be noted that components of the method(s) should be appropriate adjusted according toobjectives, conditions, and contexts.

\section{- Sources of information and data}

Information and data for CVA mainly come from secondary (available) sources as well asprimary ones (surveys, questionnaires, synthesis, analysis, calculations). Depending on CSO's operational areas, the most popular locations for CVA application are in the regions where are vulnerable to climate change and natural disasters. These localities were identified through scientific and practical evidences because they are very sensitive to climate change factors. They are vulnerable and suffered many risks, as well as losses in life and production; Climate change and sea level rise scenarios (2009, 2012 and 2016) have predicted high risks in the next 100 years for these areas .

\section{- Participants in CVAs and gender equality}

Among CSO's strengths and priorities in CVA is the attraction of many stakeholders to take part in the implementation and support process. Most CVAs are implemented at the grassroots level and some at the commune, district, and provincial levels. Whilst HVCA is widely applied at the community (village, hamlet) and commune level, PVCA is more popular at the commune and district level. CVCA, meanwhile, is adopted in a more balanced manner at all levels. Although several organizations (including World Vision, SNV, GIZ, SRD, MCD, Plan, AMDI, CRD, WWF and others) managed to get district officials involved. Their participations are mainly related to monitoring and facilitating working process with people at the commune and hamlet level. CVCA implementation mainly lies in the hands of consutants, project officers, commune officers who provided information and feedback. Local social organizations act as important partners, as main force working with CSOs and as collaborators during CVA implementation. They are Vietnam Red Cross Society, Women's Union, Farmers' Union, and Youth Union. Schools are an object for climate risk assessments, so students and teachers also take part in CVAs and are instructed to create plans for safe learning environment themselves. CSOs like Plan, World Vision, and Live and Learn have excelled in their CVA activities applied for schools.

\section{- Ensuring gender equality}

The following aspects are mainly considered: who are most vulnerable to climate change; Men's and Women's participation in CVA implementation; and each gender'svoice and feedback in decision making or adaptation planning. Vulnerable groups: Women in many rural areas in Vietnam are most affected by climate change impacts, including natural disasters. They have limited disaster response skills. This is more common among women aged above forty-five. Consequently, following CVAs, many CSOs supported women in focusing on knowledge enhancement and livelihood development. Men's and Women's participation in CVA implementation: Over $90 \%$ of surveyed CSOs said that both men and women were encouraged to take part in CVAs at the district and commune level.However, there is a clear distinction in the proportion of men and women participating in CVAs at different levels: At district and provincial level: Men's participation in CVAs accounts for an approximate $80 \%$, they are mainly support project officers. They make connections to localities, monitor the implementation process, and take part in interviews; Opposite, Women's participation is greater at the grassroots (commune, hamlet) level. They are ordinary people and female officiers who work in social organizationssuch as: Women's Union, Farmers' Union, Youth Union,... (Fig. 2). As a result, insome occasions, adaptation plans implemented by local authorities are not totally suitable to meet the demands and resources of vulnerable groups. The expectation of CSOs is that women in districts and provinces will have a greater and deeper partici- 
pation in CVA-related training courses, adaptation recommendations and plans, as well as in the supervision and assessment of the adaptation implementation process.

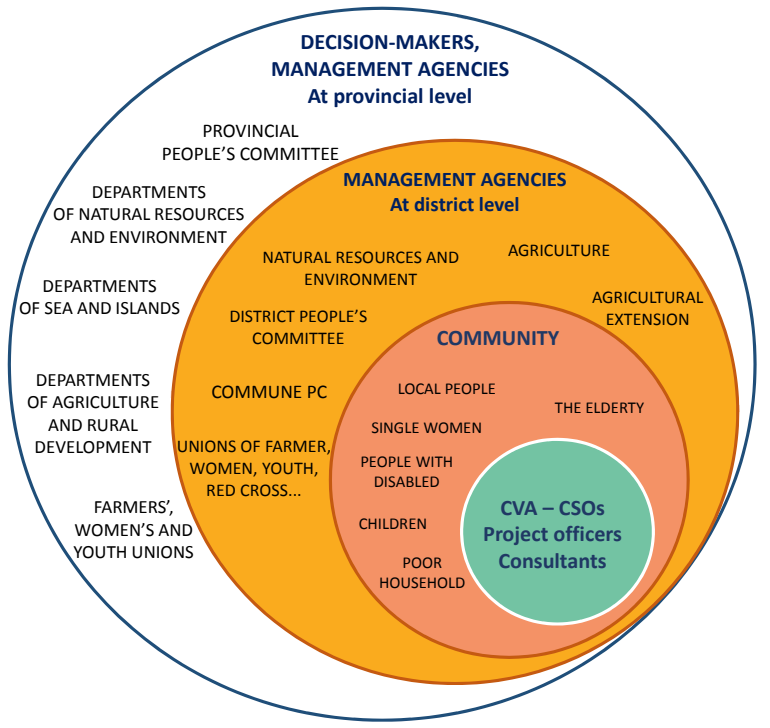

Fig. 2. Participation of local stakeholders in the implementation of CVA

\section{- Relevance to local context}

Self-assessment results show that the relevance of each method related to the local context at three levels (Irrelevant, Relevant and Highly Relevant) is rated mainly between"Relevant" and "Highly relevant". CVCA and HVCA have the highest percentages of "Highly relevant" (at $80 \%$ and $60 \%$, respectively), followed by PVCA, RAP, and VA-EbA with over $50 \%$. For methods that were assessed as "Relevant", the reason is that these methods are qualitative.if they are combined with methods/tools using local meteorological parameters such as rainfall, temperature, etc., the higher levels of relevance will be achieved.

\section{- Significance of changes from CVAbased models, solutions}

For whether the CSOs' CVA based models and solutions can lead to the expected changes or not, the Flowing Forward and VAR approaches are scored at $100 \%$ as "Very significant". The changes created by VA-EbA were assessed as "significant" and "very significant" at a rate of $50 \%$. In particular, the Flowing Forward, VAR and CVA methods in the agricultural value chains are evaluated to bring about "Very significant" changes but the actual number of CSOs implementing the method is limited, possibly due to the high demand for secondary data from specialized agencies. Many CSOs (such as MCD, AMDI, CECR) have introduced initiatives to support coastal districts to self-develop district-level adaptation action plans with the active participation of specialized departments and expert technical guidance. This is also a way to improve the capacity of local officials.

\section{- Impacts/Number of beneficiaries}

Most CSOs say it is difficult to collect the number of CVA beneficiaries' localities as communities are very diverse, scattered throughout the climatic regions of Vietnam or forecasted to be vulnerable to current and future climate change. Some typical examples such as: The IRRI Institute - CCAFS uses the PVCA method to benefit households in 13 Mekong Delta provinces; The 5-year HVCA application by World Vision benefits roughly 10,000 people. In communities and communes where CVA is applied by World Vision, beneficiaries are mostly women (about 65\%) and the poor (about 80\%); CARE International uses HVCA and CVCA methods for around 20 years to help thousands of people (specific data is not available) across Vietnam's three regions (the North, the Centre and the South); SNV, Plan, GEF, SRD, MCD, WWF, CRD and others conducted CVA integration in more than 15 provinces, and enabled many districts, communes with thousands of people in mountainous, coastal and desert areas to cope with natural disasters and to improve their livelihoods.

\section{- Effectiveness of CVA implementation in 7 priority sectors of the NAP.}

The sectors in which CVAs are conducted by CSOs are relatively similar to the seven priority sectors of the NAP. They are Water, Agriculture, Land, Biodiversity, Transportation, Gender and 
Poverty Reduction, and Healthcare and Education. However, CVA methods are applied at different levels in each sector (Table 1).

Table 1. Summary of CVA methods applied by CSOs in priority sectors in Vietnam's NAP

\begin{tabular}{llll}
\hline Sector & \multicolumn{3}{c}{ Common CVA methods in } \\
priority sectors
\end{tabular}

As shown in Table 1, HVCA is the most popular methods. According to the statistics, more than $50 \%$ of the interviewees applied HVCA, PVCA seconds with 42,9\%, third being CVCA (43\%) and fourth being VA-EbA (14\%).

Adaptation measures that are applied as a result of CVAs implementation

CVA implementation and results are applied most in the agro-forestry-fishery sector, medicinal plant development closely connected with preservation and livelihoods, and husbandry accompanied by technical improvements. There are many adaptation models, solutions and practices that refer to and use results from CVA, mainly in livelihoods, resource management (water, biodiversity, land use), education, and lowering floors, environment, health. CVA have been developed and used in more than 30 provinces. It is used as an input for design and implementation of livelihood interventions in the direction of a livelihood-centered approach. This is because at community and household levels, livelihoods are most affected by and vulnerable to climate change and are the greatest concern and need of households. Promoting adaptive livelihoods will mitigate vulnerability and build up community resilience. Fig. 3 summarizes a number of local stakeholders where CSOs play central role in climate vulnerability assessment and then chair or coordinate implementation of climate change adaptation models in the priority sectors of the NAP.

\section{CSOs' capacity and roles in CVA implemen-} tation

The survey results show that CSOs are confident in the CVA implementation at local and community level and that CVA results have made significant contribution to local adaptation capacity. Most interviewed organizations (57\%) hire consultants to support local officials and assessment projects. $37 \%$ of CSOs actively work with officials and collaborators to conduct CVAs, and a few of them rely completely on external consultants for assessment processes. CSOs' strengths in CVA implementation: (1) They adopt a community-based approach based on a community perspective, mobilize the participation of local people and other stakeholders; focus on disadvantaged and vulnerable groups, develop adaptation solutions that generally meet the needs and capabilities of the population while being in line with the local natural context and policies; (2) CSOs have shown great performance in raising awareness and in building local capacity via organizing training courses, conducting community communication, and creating favorable conditions for local people to participate in projects' activities; (3) They are also flexible in method application, adopting quantitative and qualitative methods involving participatory tools. Collaborators are trained in advance and are local residents so information collection is facilitated. Collected information is helpful as it is diversified, embeds history, truly reflects the current vulnerability status, and receives verification and on-the-spot feedback, 4) CSOs appreciate indigenous knowledge and often actively compile this knowledge and experiences of different communities, which enables them (to have important information as a reference for future recommendations which are in line with local demands, capacities, and cultural practices, (5) Thanks to their reputation of maintaining flexible working mechanisms and transparent finance processes, CSOs can easily gather groups of experts, technicians and scien- 
tists of different sectors and competences and enthusiasm into play in assessment activities as well as insight into methodologies. CSOs are also experienced in effectively organizing multilateral group work, ensuring good results, timeline, and cost saving needs, (6) CSOs are typically transparent and proactive in sharing information and results of both CVAs and projects with stakeholders (authorities, specialized agencies, scientists, and communities) as well as with other CSOs and localities. As a result, the CSO network in Vietnam will likely expand, helping to extend the benefits of CVAs while saving time and human resources during the implementation of new CVAs.

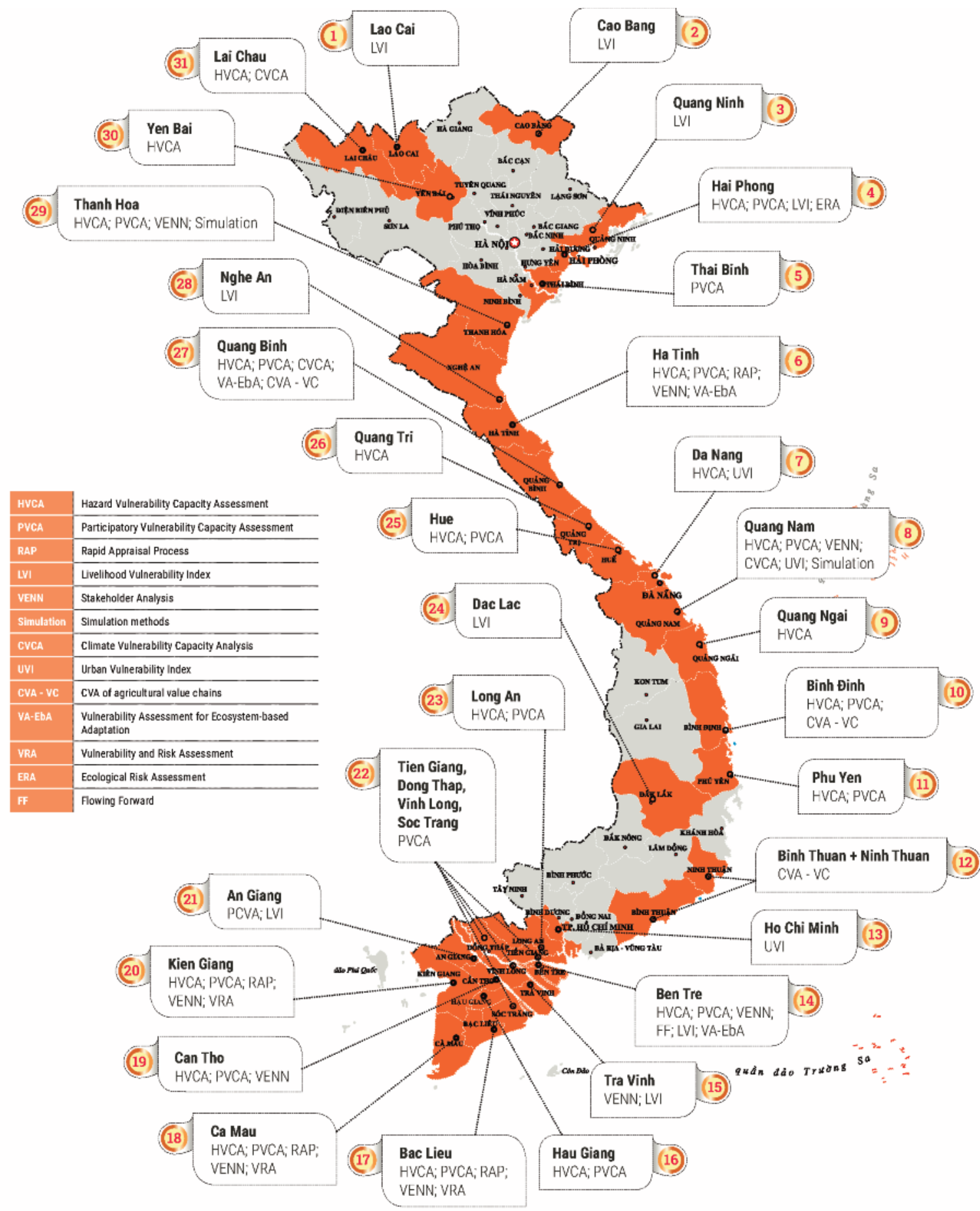

Fig. 3. CVA areas applied by CSOs in Vietnam 
Despite its own advantages and strengths, the implementation of the CVA still faces difficulties in data sources, capacity of local officials and information channels of CSO network:

(1) Information and data: CSOs state that they have difficulties getting access to official updated sources of information and data by specialized agencies during CVA implementation. For this reason, input data is insufficient for the assessment of potential impacts and risks in the long run. Limited access to specialized database sources makes assessment results more quantitative than qualitative and there are limitations regarding CVA long-term forecasts on local potential climate risks. It also makes it hard for CSOs to convince local authorities and specialized agencies to integrate CVA results in their development plans;

(2) CVA human resource: Direct engagement of officials from specialized agencies at the provincial and district level is limited resulting in a lack of monitoring and correction of the accuracy of the assessment results, and affecting the CSO's goal of "enhancing community participation to improve adaptive capacity".

(3) Information sharing of CSOs: Currently, the network of NGOs in Vietnam has not yet established and maintained a stable, complete and updated database about activities that CSO has been implementing. Therefore, restricting the sharing, exchanging information among CSOs as well as limiting timely and formal information delivery from CSOs to government agencies, partners, and sponsor.

(4) CVA for urban areas: There are currently few vulnerability assessments conducted by CSOs for climatic risks in urban areas such as flooding, heat stroke, prolonged heat stress causing environmental risks, health and transportation. The main reason is due to the lack of technical, financial and data capacity.

\section{Conclusions and recommendations}

\section{Conclusions}

CVAs implemented and supported by CSOs have brought about significant results, helping local communities and authorities effectively develop and implement adaptation plans and solutions. At the provincial level, disaster-climate change-related information and events as well as indigenous knowledge, which is acknowledged, collected from stakeholders and analyzed is of high credibility and practicality.

With a point of view to enhancing the communities' voices and ability to take action, CSOs in Vietnam are implementing vulnerability and adaptive capability assessment methods which pave the way for interventions as well as for adaptive support and promote the integration of climate change factors into development planning and plans.

The CVA results are important, practical and up-to-date input information for adaptation plans and solution development in localities; Authorities, sectors, development organizations and households can easily use and integrate the results from CVA for climate change adaptation goals.

CVA helps strengthen the knowledge, awareness, skills and responsibilities of stakeholders at all levels regarding climate change response and sustainable development.

Gender issues have been emphasized in CVAimplementation and women have participated. The voices and aspirations of women and girls in CVAs are encouraged and promoted. Many adaptive models that were implemented after the CVAs significantly improve women's lives and reduce their vulnerability.

Regular CVA reviews (annually or every 5 years) are of great significance for annual socioeconomic plan development of localities.

CSOs have not implemented CVA in urban 
areas. They also face difficulties when information quality from assessment are still poor due to limited capacity of each $\mathrm{CSO}$, financial resources and other reasons.

\section{Recommendations}

To supportthe government to enhance the use of CVAs in the NAP implementation after 2020, the following recommendations are put forward:

Mechanisms and policies: The CVA implementation in localities should be institutionalized in national documents to create a legal status for periodic or annual CVA implementation. CVAs should be considered as a necessary requirement for projects, planning and development plans to ensure a timely review of current and potential climate risks which allows for preventive response measures in line with the local capabilities. The application and integration of CVA results should be systematically enhanced into planning at all levels, and in all sectors and priority areas of the NAP.

CVA implementation in climate change projects: The combination of bottom-up approaches, top-down approaches and inter-sectoral, ecosystem-based approaches should be enhanced in CVAs. Local authorities at the provincial and district levels need to participate more actively in the whole process of CVA.

CVA methods, information, data and area expansion: The authorities and specialized agencies need to be more open regarding their databases, materials and information sharing as well as generally giving access to CSOs and the communities. The CSOs network needs to build and maintain website information, database of CSO activities and results.

CVA areas: Climate Vulnerability Assessments in large urban areas need boosting, especially in densely populated areas that are facing increased flooding and heat impacts due to rising temperatures. Such directly impacts of environment, health and transportation are under prior- ity areas of the NAP.

Gender equality: The participation of vulnerable groups, including women, should be ensured and enhanced in the NAP process. Gender segregated data should be used in vulnerability and adaptation assessments. Training on a general understanding of gender should be provided for parties in charge of the NAP development and implementation.

Information exchange and communication: Multi-lateral information exchange take place among parties, including local authorities, leaders, CSOs, scientists, communities, and the private sector on CVA implementation and application of its results. Information needs to reach the majority of the population, specialized officials, as well as local leaders. It is also necessary to facilitate business involvement in CVA assessments and feedback processes and reference of CVA results in their investment decisions to minimize risks.

International cooperation enhancement: The Department of Climate Change, CSOs and CCWG need to enhance their cooperation with international partners in terms of pushing forward CVA in NAP development and implementation. This should be done together with diversifying mechanisms and sectors: recommended areas include information and data sharing, fundraising, joint-implementation and monitoring and evaluation.

\section{Acknowledgements}

Thanks to the Friedrich-Ebert-Stiftung Institute (FES), Germany and the Vietnam Climate Change Working Group (CCWG). This paper uses some information and data from the Advocacy Orientation Research Report on "Climate Vulnerability Assessments by Civil Society Organizations Towards the National Adaptation Plan (NAP) In Vietnam" by the authors them- 
selves with financial support from FES and CCWG, completed in 2019.

\section{References}

1. Angie, D., Kaia, A., Charles, E., 2009. Climate Vulnerability and Capacity Analysis Handbook, CARE international.

2. Doswald, N., Osti, M., 2011. Ecosystembased approaches to adaptation and mitigation good practice examples and lessons learned in Europe. Federal Agency for Nature Conservation, Germany.

3. Füssel, H.M., Klein, R.J.T., 2006. Climate Change Vulnerability Assessments: An Evolution of Conceptual Thinking. Climatic Change, 75(3): 301-329.

4. Hoang, T.N.H., Truong, Q.H., 2016. Development to climate change adaptive Livelihood by Ecosystem - based Approach (EbA). Vietnam Natural resources and Environment Magazine, 4: 7-10.

5. Hoang, T.N.H., Nghiem, T.P.T., Truong,
Q.H., 2019. Report of Climate Vulnerability Assessment by Civil Society Organizations: Towards the National Adaptation Plan (NAP) in Vietnam. Printed by FRIEDRICH EBERT STIFTUNG and Climate Change Working Group in Vietnam, Hanoi, 2019.

6. Le, N.T., 2017. Research overview of climate change vulnerability assessment. Journal of Science and Technology Development, 2: 5-20.

7. MARD and UNDP, 2015. Communitybased Disaster risk Assessment Guidelines, Disaster Management Center, Hanoi.

8. Truong, Q.H., Hoang, T.N.H., Vu, T.B.H., 2019. Booklet "Civil society organizations and the National Plan for Climate Change Adaptation". UNDP/GEF SGP and SRD, Hanoi.

9. TTCP, 2016. Plan for implementation of the Paris Agreement by Decision No. 2053/QDTTg.

10. UNFCCC, 2015. Adoption of the Paris Agreement. Conference of the Parties, Twentyfirst session, Paris. 